\title{
Intrauterine Infection: Pathological Study on Chorioamnionitis and Villitis of Unknown Etiology
}

\author{
Hiroshi ITOH, KeISUKe HANIOKA, ChIHo OBAYASHI, NAOKI KANOMATA, \\ KYOKo ITOH, YUKIHIRo IMAI, NAOTO KURODA AND YoshitaKe HAYASHI, \\ The First Division of the Department of Pathology, \\ Kobe University School of Medicine, Kobe 650, Japan
}

\begin{abstract}
Twenty-five placentae between 24 and 41 gestational weeks showing signs of intrauterine infection were studied pathologically. The placentae with chorioamnionitis at less than 33 gestational weeks had thick amniotic layers due to infiltration by polymorphonuclear leucocytes (PMNL) and fibrin deposits. Umbilical cords also showed signs of infiltration and occasional calcification. The main cause of chorioamnionitis in these cases is ascending bacterial intrauterine infection had villitis with cholangiosis and dysmature villi in 6 placentae. The clarification of these infectious processes is difficult, and they are mostly diagnosed as villitis of unknown etiology (VUE). Autopsy data are useful for the clarification of infectious pathology in stillborn cases.
\end{abstract}

Key words: Intrauterine infection, Chorioamnionitis, Villitis of unknown etiology

(Endocrine Journal 41(Suppl): S135-S140, 1994)

INTRAUTERINE infection ascends from the endocervical canal, or reaches the placenta hematogenously through the maternal blood. Rarely it is acquired by amniocentesis, sampling from chorionic villi, amnioscopy, percutaneous umbilical blood sampling, or intrauterine fetal transfusion [1]. Syphilis, listeriosis, toxoplasmosis and cytomegalovirus infections are known to cause hematogenous placental infections [2], but they are rapidly decreasing in number in Japan. Villitis of unknown etiology (VUE) is closely correlated to IUGR and stillbirth and other conditions [3, 4]. Among the intrauterine ascending infections, amniotic infection occurs at first, and then causes turbid amnionitis followed by polymorphonuclear leucocytes (PMNL) infiltration, with discolouration and edema of the umbilical cord. The degree of placental surface turbidity correlates to the severity of the maternal infection. Pathological examination of the

Correspondence to: Dr. Hiroshi ITOH, Department of Pathology, Kobe University School of Medicine, 7-5-1 Kusunokicho, Chuoku, Kobe 650, Japan placenta is useful in investigating the maternal effects and IUGR and still birth, in addition to chorioamnionitis and VUE.

\section{Materials and Methods}

Twenty-five placentae between 24 and 41 gestational weeks were examined pathologically. Fourteen babies delivered at less than 33 gestational weeks were suffering from fetal distress, and 5 were stillborn. Six placentae at full term were all from stillborn which were thought to be infected in utero. These placentae were fixed in $10 \%$ formalin solution, and processed routinely in dehydration to paraffine blocks, then cut into 4 $\mu \mathrm{m}$ thick serial sections. Histological sections were stained with hematoxylin eosin. Immunohistoenzymatical stains for IgG, ASD-CI LCA, herpes virus 1 and 2, hepatitis virus, and cytomegalovirus in a few cases, were done by peroxidase anti-peroxidase methods. Microbiological or other viral examinations were not used in these placentae. 


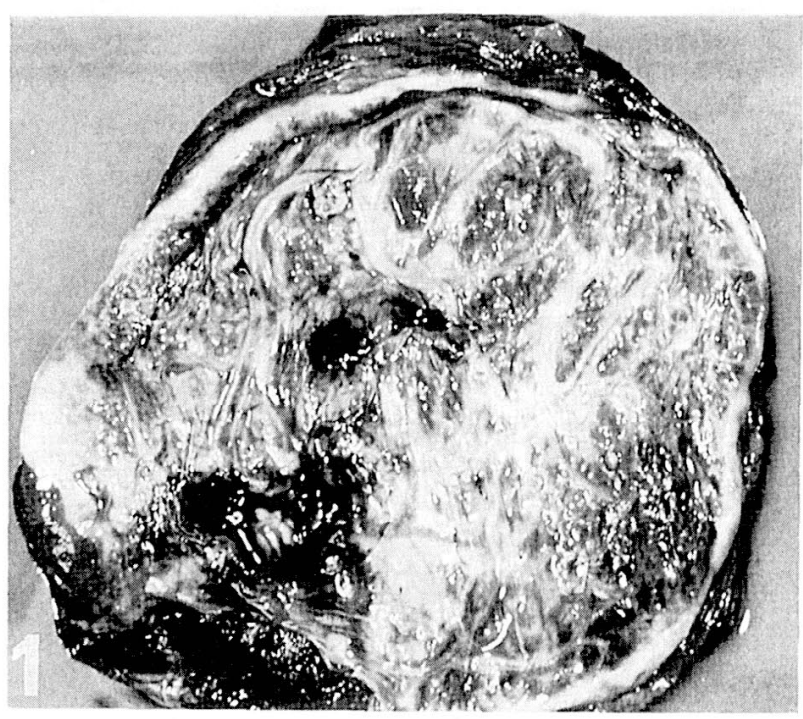

Fig. 1. Severe chorioamnionitis showing grayish-yellow surface of the placenta at 29 weeks of gestation.

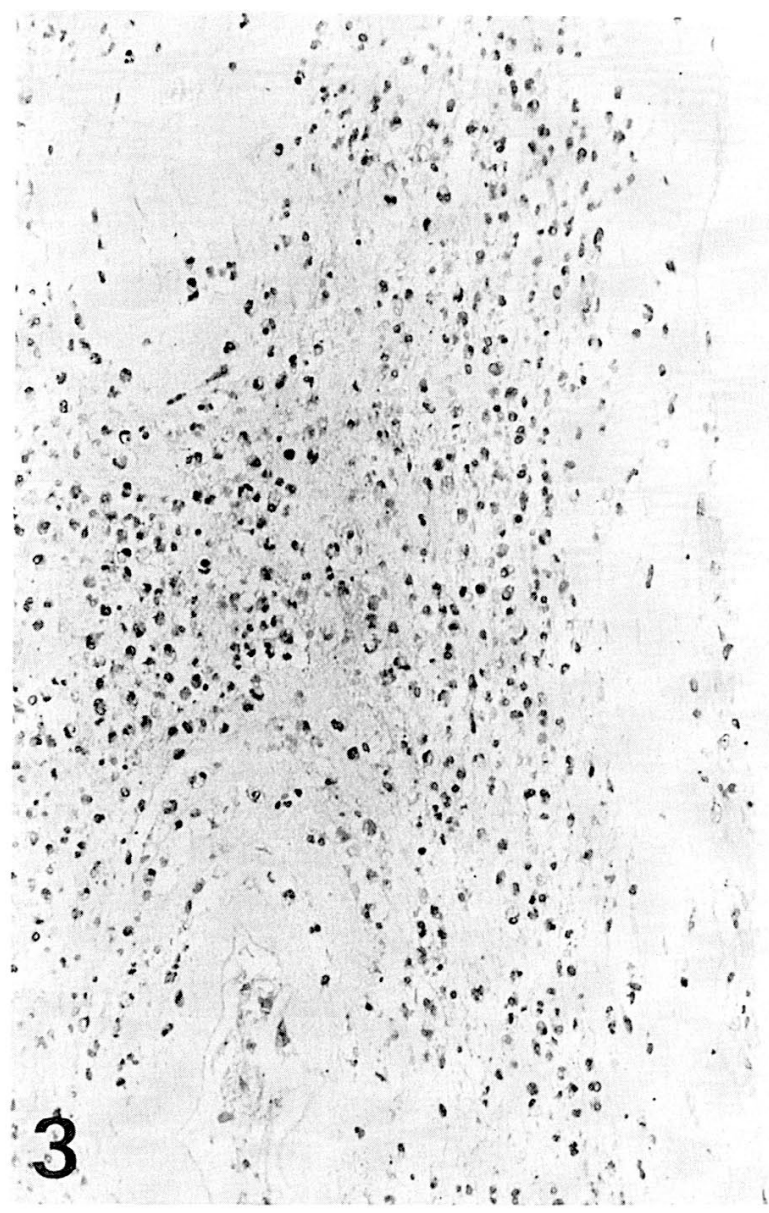

Fig. 3. Severe chorioamnionitis with numerous polymorphonuclear leucocytes (PMNL) infiltrating at 29 weeks of gestation, ASD-CI stain, $\times 200$.

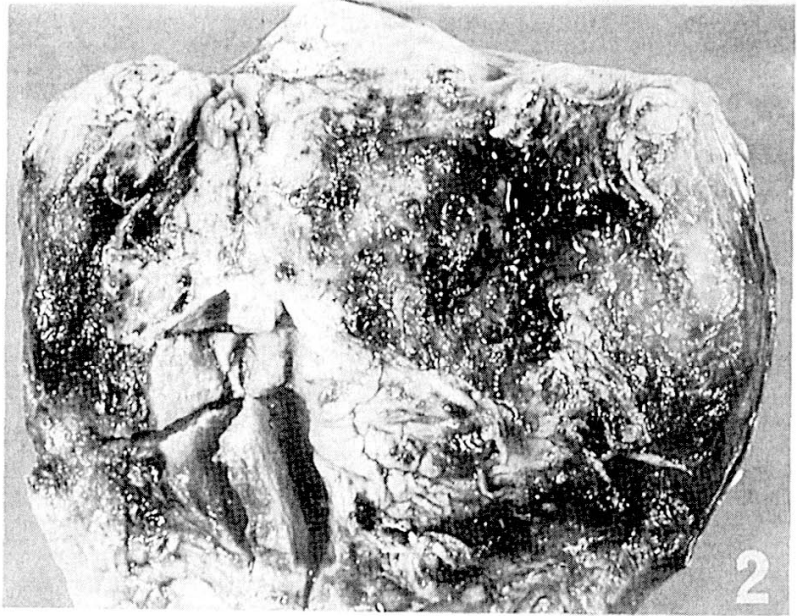

Fig. 2. Severe deciduitis with early abruptio placenta at 40 weeks of gestation.

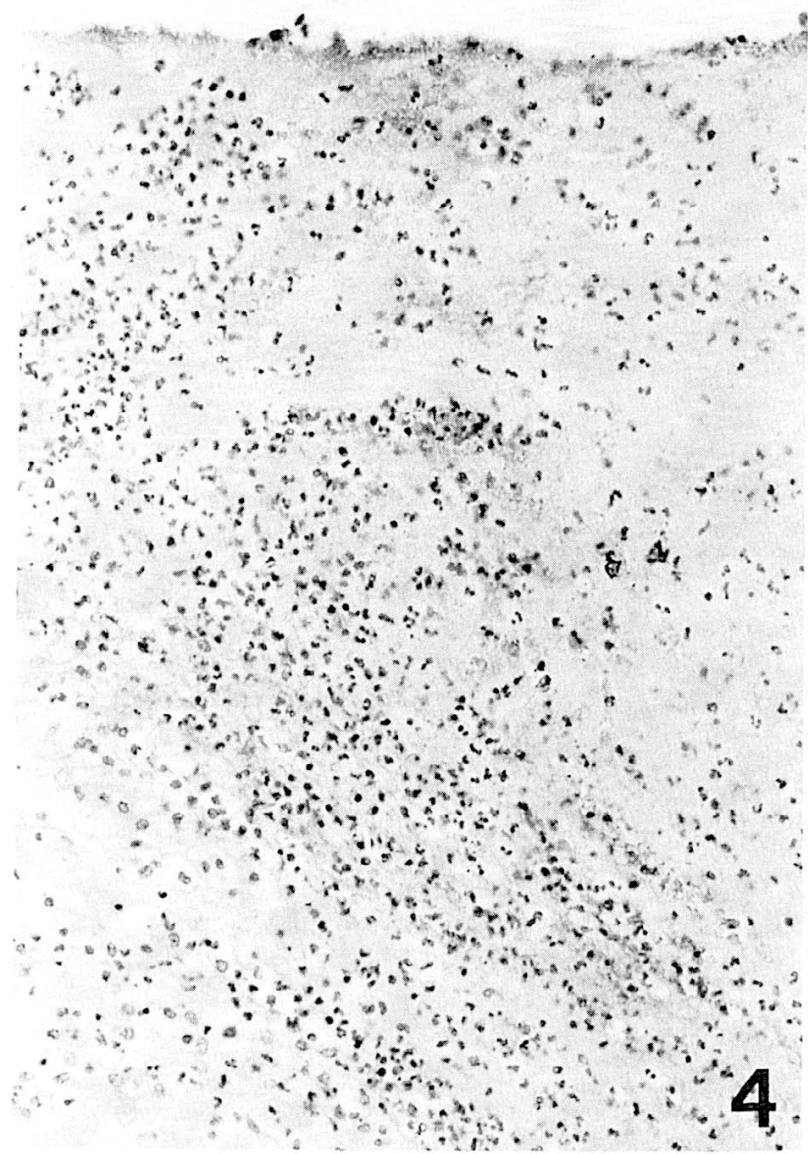

Fig. 4. Severe chorioamnionitis with numerous PMNL and amnion necrosis at 31 weeks of gestation. IgG stain, $\times 200$. 


\section{Results}

Macroscopic figures of the placentae between 24 and 33 weeks of gestation showed signs of marked chorioamnionitis in all cases examined. The amnionic surface is turbid and yellow with adhesion to the chorionic membrane (Fig. 1). On the other hand, the amnionic surface of the inflamed. On the decidual side of the placentae there was deciduitis and hematoma sometimes associated with abruptio placenta (Fig. 2).

\section{A. Chorioamnionitis in the placentae between 24 and 33 weeks of gestation}

The histological features of chorioamnionitis are a thin to thick amnionic layer caused by the dif- fuse infiltration of PMNL and fibrin deposits. ASDCI stain was specifically positive in the cytoplasms of PMNL (Fig. 3). IgG was also positive in the cytoplasmic membrane of PMNL and in the stroma of the amnionic layer (Fig. 4). The cytoplasms of PMNL were positive or faintly positive. Infiltrating cells were mostly composed of PMNL. These inflammatory foci easily cause further dissemination; for example to the umbilical cord, in which calcification is formed in the later stage of inflammatory process. Many PMNL infiltrate the wall of the umbilical artery (Fig. 5), and the vein or the surrounding Wharton's jelly, forming socalled funisitis. Severe chorioamnionitis occasionally causes diffuse and prominent villitis with many PMNL. Figure 6 shows villitis caused by lymphocytic infiltration. Although the stillborn baby in this case was not thought to have herpes virus in-

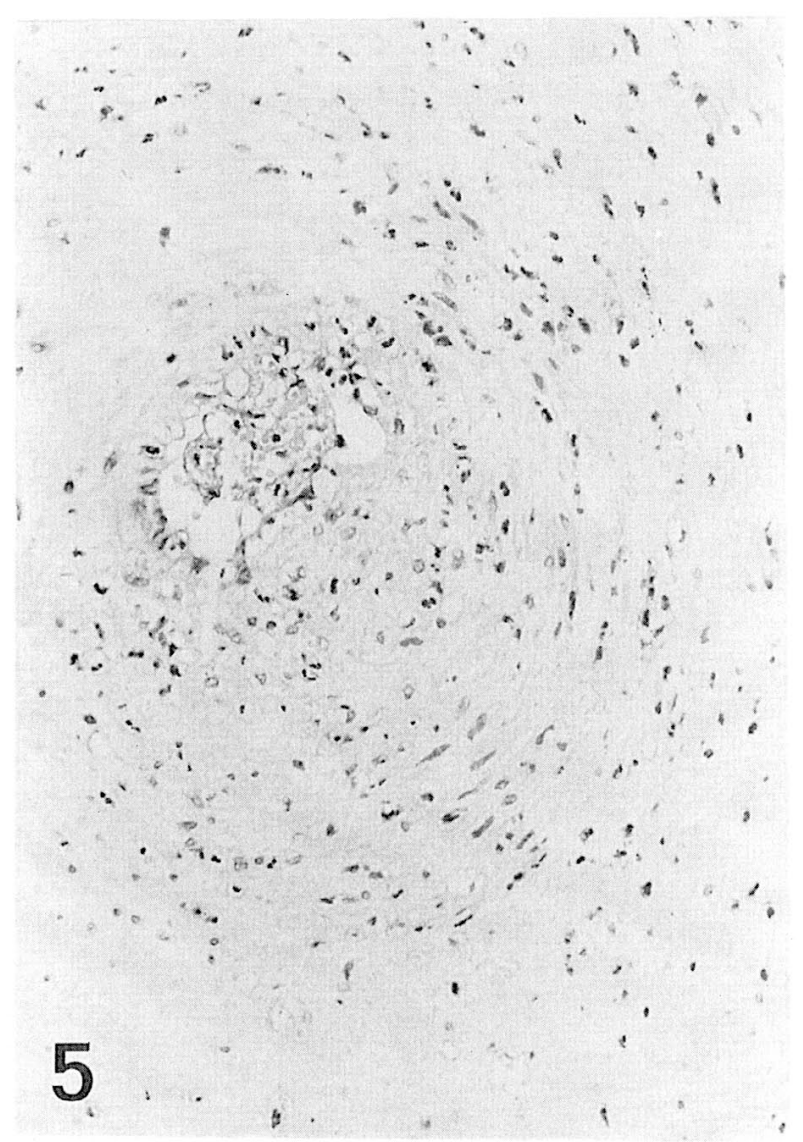

Fig. 5. Funisitis with many PMNL in the wall of an umbilical artery, 32 weeks of gestation, ASD-CI stain, $\times 200$

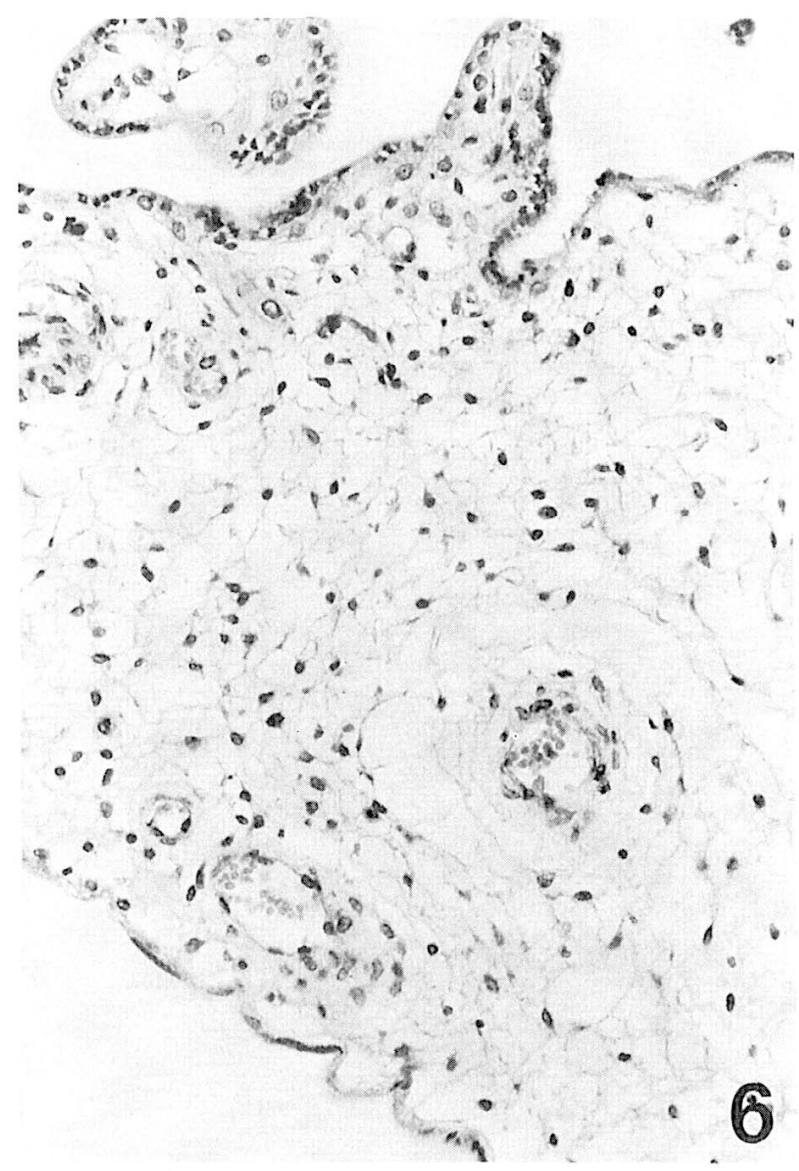

Fig. 6. Villitis showing lymphocytic infiltration in the edematous stroma of the chorionic villi at 24 weeks of gestation, LCA stain, $\times 200$. 
fection, the chorionic villi contained neither viral inclusion bodies nor positive cells in herpes virus stains.

\section{B. Villitis of the placentae at full term}

Villitis of the placentae at full term is rare and not generally very severe. The histological characteristics are cholangiosis and immaturity of the villi. In cholangiosis there are more than 10 capillary vessels in a villus, that is, capillary hyperplasia in a villus. A few PMNL in a villus were dispersed in the villous interstitium and positively stained with ASD-CI (Fig. 7). Dysmature villi have a distorted or anomalous sharp, just like the sweets leaves of a "Yatsude" plant or Japanese old confectionery "Konpeitoh". Figure 8 shows PMNL in the villous stroma positively stained with IgG. Two cases among 6 placentae examined at full term showed marked deciduitis associated with abruptio placentae. These had foci of many PMNL in the intervillous space around the deciduitis (Fig. 9). Some foci resembled a degenerative villus with many PMNL, fibrin deposit and red blood cells delineated by fragments of the capillary basement membrane (Fig. 10). In some cases viral infection was unlikely in view of the maternal clinical data, but all examinations were hepatitis virus and so forth.

\section{Discussion}

Pathological diagnosis of chorioamnionitis in the earlier weeks of gestation is easy macro- and microscopically. The main cause of this ascending

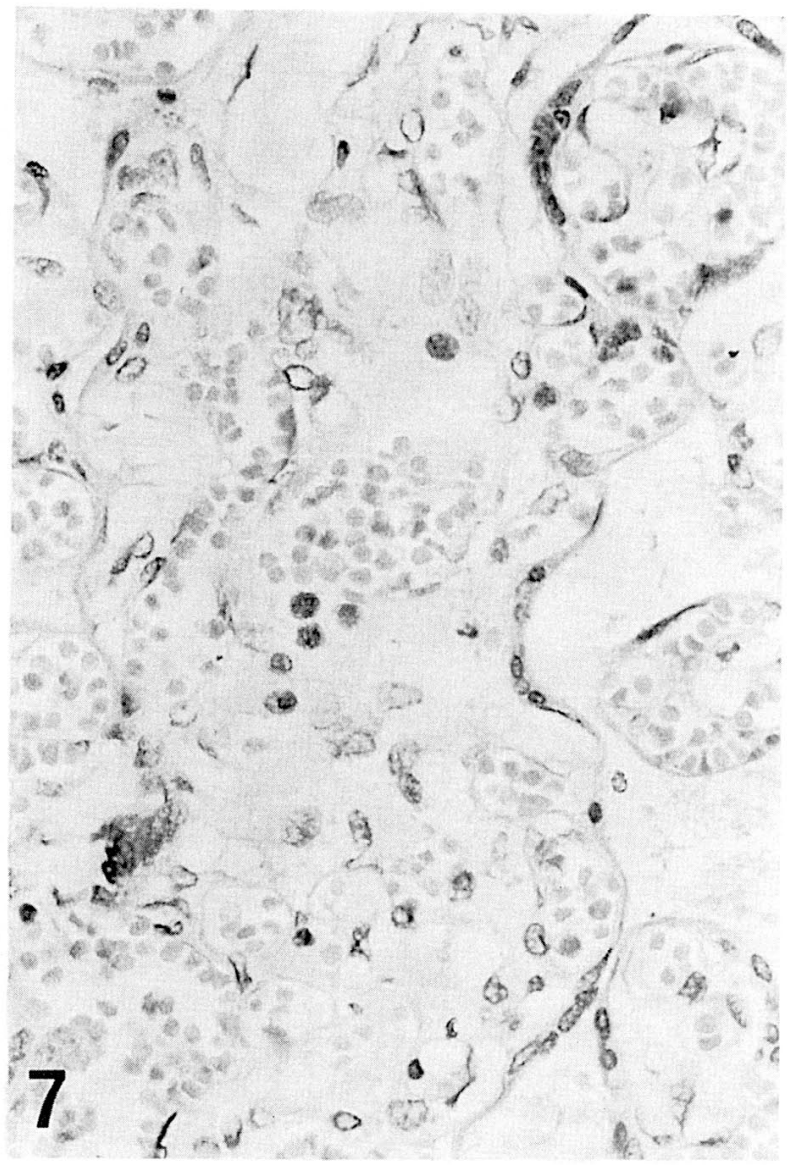

Fig. 7. Villitis showing a few PMNL in the chorionic villi at 40 weeks of gestation, ASD-CI stain, $\times 400$.

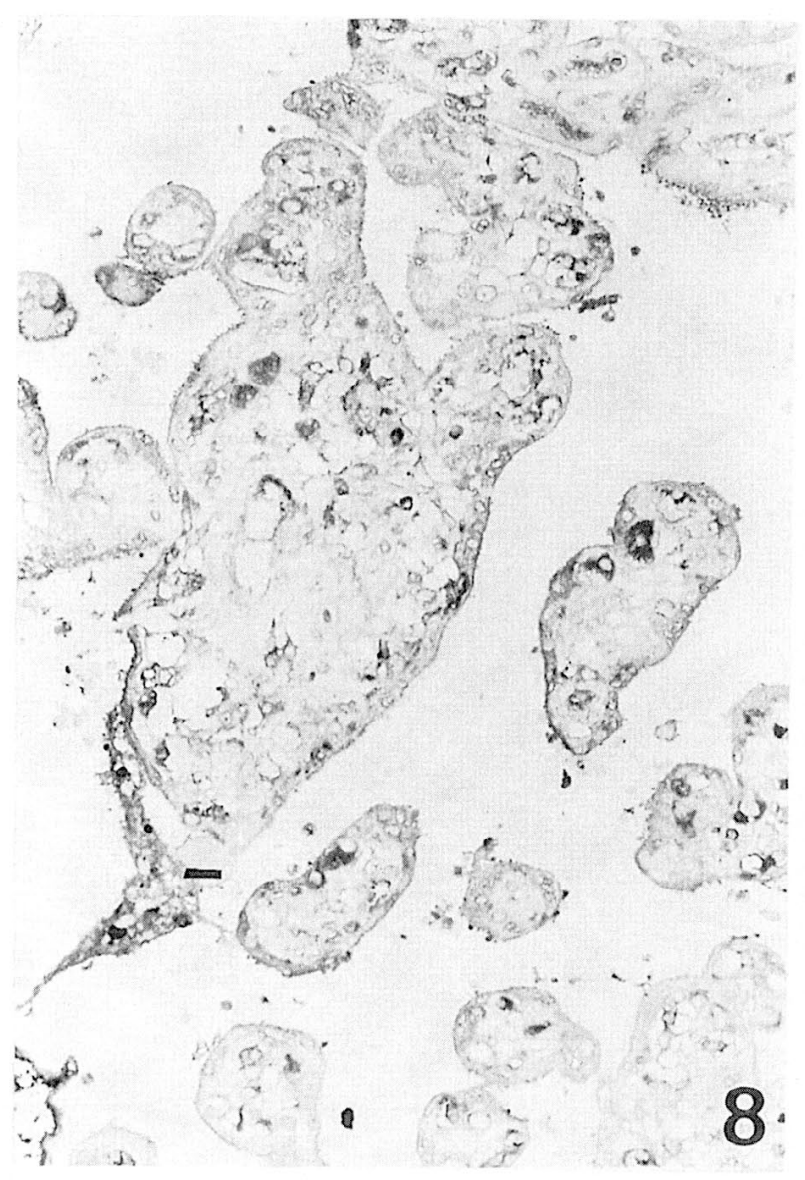

Fig. 8. Villitis showing a few PMNL in the chorionic villi at 41 weeks of gestation, IgG stain, $\times 200$. 
chorioamnionitis is from bacterial infections such as gardnerella vaginalis, ureaplasma urealyticum and others.

Blanc et al. reported umbilical cord infection as the name of "Subacute necrotizing funisitis", which may closely correlate to the occurrence of WilsonMikity syndrome [6, 7].

Although congenital syphilis, listeriosis, toxoplasmosis and cytomegalovirus infection are rare in Japan, these are now seen in developing countries. Congenital syphilis of the placenta results in extensive necrosis with necrotizing angitis and proliferative inflammation. There are PMNL and lymphocytes and a few epithelioid cells with vasculo-connective tissue in the necrotic region [8]. In listeriosis, there are foci of whitish-yellow abscess in the amnionic membrane. The Listeria monocytogenes is positively stained with gram

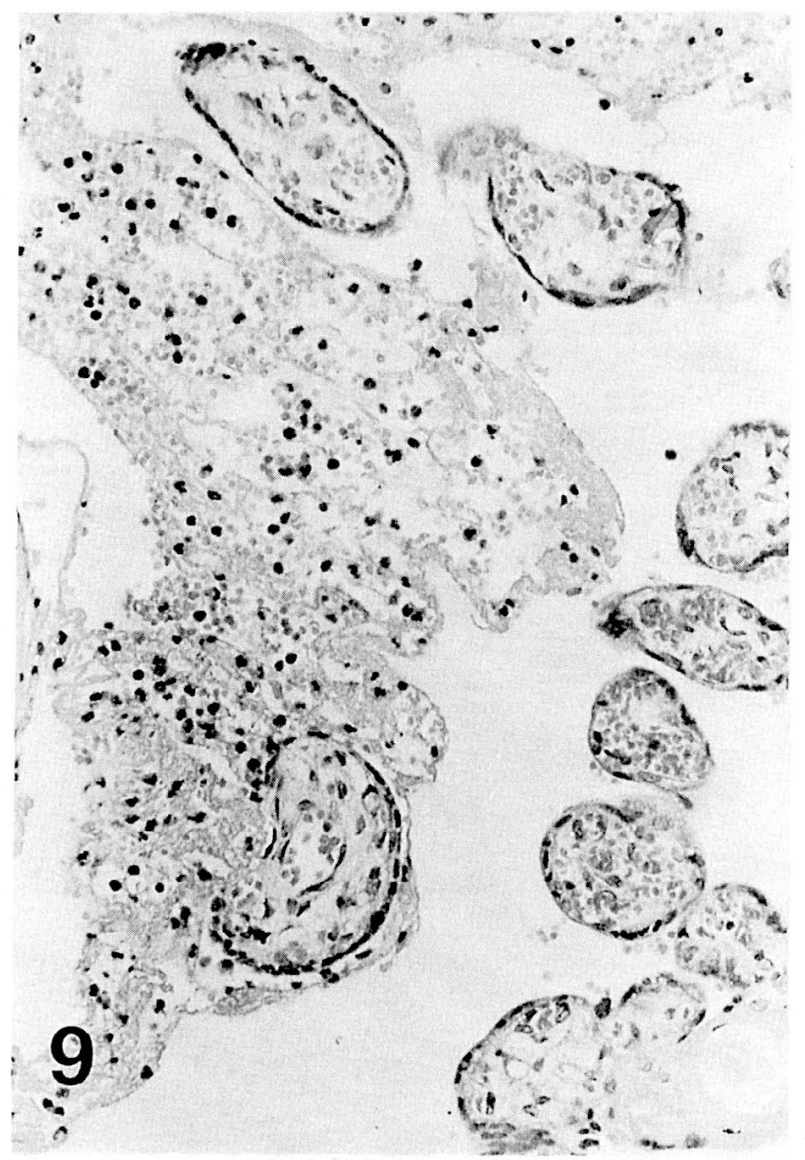

Fig. 9. Many PMNL in the intervillous space at 39 weeks of gestation, ASD-CI stain, $\times 200$. stain [9]. Cytomegalovirus inclusion bodies are found in the lungs, kidneys, liver and elsewhere in the infected stillborn. The placenta infected by cytomegalovirus reveals plasma cell villitis with a few inclusion bodies, when carefully examined by the pathologist [10].

It is difficult, however, to diagnose mild chorioamnionitis in the placentae at full term. Differential diagnosis must take into account old hemorrhage and meconium deposits on the amnionic membrane. Histological examniation is also necessary in cases with a discoloured amnion surface. Intervillous PMNL around severe deciduitis associated with abruptio placentae may cause perinatal or postnatal infection of the neonate. It is necessary to preserve such a placenta without fixation for a few days by keeping it in a refrigerator, and to examine it bacteriologically and pathologically af-



Fig. 10. Many PMNL and fibrin at 40 weeks of gestation, ASD-CI stain, $\times 400$. 
ter the occurrence of the infection in the neonatal period.

Pathological examination of villitis of unknown etiology (VUE) is difficult but necessary to clarify the pathogenesis of stillborn and IUGR babies, and to devise effective treatments for them as soon as possible.

\section{Conclusion}

Intrauterine ascending infection is induced by chorioamnionitis and funisitis. The degree of turbidity and discolouration, and of PMNL infiltration in the amnionic membrane is compatible to the severity of maternal infection. Though VUE at full term is defficult to examine, pathological study on VUE is useful for the pathogenesis of stillborn and IUGR babies correlated to maternal effects.

\section{Acknowledgment}

We wish to thank Dr. Masahiro Nakayama, Department of Pathology, Osaka Medical Center and Research Institute for Maternal and Child Health, for his great help and advice. We are also grateful to Dr. Tsunehisa Makino, Department of Gynecology, Keio University School of Medicine, for his kindness.

\section{References}

1. Benirschke K, Kaufman P (1990) The Placenta, Infectious Disease. Springer-Verlag. New York, pp 542-559.

2. Fox H (1978) Pathology of the Placenta, Infection of the Placenta. W. B. Saunders Camp. Ltd., London, pp 286-325.

3. Garcia AGP (1982) Placental morphology of lowbirth weight infants. In: Soma $\mathrm{H}$ (ed) Morphological and Functional Aspects of Placental Dysfunction. Karger, A.G. Basel, pp 100-112.

4. Wakayama $Y$, Nakayama M, Imai S, Takeuchi $T$ (1989) Villitis of unknown etiology in sibling cases. Symposium on Perinat Med 7: 33-38 (In Japanese).

5. Liechty KW, Koenig JM, Mitchell MD, Romero R, Christensen RD (1991) Production of interleukin-6 by fetal and maternal cells in vivo during intraamniotic infection and in vitro after stimulation with interleukin-1. Pediatr Res 29: 1-4.

6. Blanc WA (1981) Pathology of placenta, membranes, and umbilical cord in bacterial, fungal and viral infections in men. In: Naeye, Kissane, Kaufman (ed) Perinatal Diseases. International Academy of Pathology Monograph. Baltimore/London, Williams and Wilkins, pp 67-132.

7. Nakayama M (1994) Clinical significance of chorioamnionitis of the placenta-especially concerning to neonatal pulmonary diseases. Pathol Clin Med 12: 417-424 (In Japanese).

8. Fojaco RM, Hensley GT, Hoskowitz L (1989) Congenital syphilis and necrotizing funisitis. JAMA 261: 1788-1790.

9. Boucher $\mathrm{M}$, Yonekura ML, Wallace RJ, Phelar JP (1984) Adult respiratory distress syndrome: a rate manifestation of Listeria monocytogenes infection in pregnancy. Am J Obstet Gynecol 14: 686-688.

10. Anonymous (1989c) Screening for congenital CMV. Lancet 2: 599-600. 